\title{
O uso de computadores de baixo custo para aplicação da metodologia de cenário de simulação em laboratório de vôo
}

\author{
The use of low-cost computers to apply the simulation methodology in \\ the flight laboratory
}

1 Margareth Hasse margareth.hasse@utp.br

1 Maurício Lorenzini Coelho

1 Universidade Tuiuti do Paraná

\section{Resumo}

Este trabalho trata da aplicação do cenário de simulação como metodologia ativa de aprendizagem nas disciplinas que compõem o currículo do Curso Superior de Tecnologia de Pilotagem Profissional de Aeronaves ${ }^{2}$. A pesquisa surge de dois questionamentos dentro da educação superior: o uso das metodologias ativas e o currículo baseado em competências. 0 estudo envolve, basicamente, o uso do computador de baixo custo em laboratório de voo onde se criam situações reais em um ambiente virtual para aplicar a teoria. Essa prática inovadora de aprendizagem baseada em cenário de simulação em laboratório de voo, integrado a outras estratégias didáticas, motiva o discente e gera nele 0 sentido de responsabilidade sobre o seu aprendizado. Além da revisão bibliográfica sobre a metodologia no desenvolvimento das competências do aluno de pilotagem de aeronaves, esta pesquisa apresenta os dados coletados sobre a evolução do uso, pelos docentes, do laboratório de voo desde a sua criação, assim como sobre a aplicação da metodologia e a percepção dos discentes do CSTPPA quanto ao seu aprendizado. O próximo passo é mensurar a qualidade do desenvolvimento das competências no aprendizado dos discentes e as diferenças na aplicação da metodologia por disciplina.

\section{Palavras-chave}

Cenário de simulação. Prática inovadora de aprendizagem. Currículo baseado em competências.

\begin{abstract}
This work deals with the application of the simulation scenario as an active learning methodology in the disciplines that make up the curriculum of the Higher Aircraft Professional Pilot Technology Course. The research arises from two questions within higher education: the use of active methodologies and the competency-based curriculum. The research basically involves the use of a low-cost computer in a flight laboratory where real situations are created in a virtual environment to apply the theory. This innovative learning practice based on a simulation scenario in a flight laboratory, integrated with other didactic strategies, motivates the student, and generates in him a sense of responsibility for his learning. In addition to the bibliographic review on the methodology in the development of the skills of aircraft piloting students, this research presents the data collected on the evolution of use by the teachers of the flight laboratory since its creation, as well as on the application of the methodology and the perception of CSTPPA students regarding their learning. The next step is to measure the quality of the development of skills in student learning, and the differences in the application of the methodology by subject.
\end{abstract}

\section{Keywords}

Scenario-based learning. Innovative learning practice. Skills-based curriculum.

2 O CSTPPA pertence à Universidade Tuiuti do Paraná e está localizado em Curitiba, no Campus do Bacacheri.

\section{Como você deve citar?}

HASSE, Margareth; COELHO, Maurício Lorenzini. O uso de computadores de baixo custo para aplicação da metodologia de cenário de simulação em laboratório de vôo. Cadernos UniFOA, Volta Redonda, n. 43, p. 63-73, agosto 2020. 


\section{INTRODUÇÃO}

O presente artigo é resultante de parte da pesquisa sobre a aplicação da metodologia ativa de aprendizagem baseada em cenário de simulação realizada pelo grupo de professores do Curso Superior de Tecnologia em Pilotagem Profissional de Aeronaves (CSTTPA), da Universidade Tuiuti do Paraná $(\mathrm{UTP})^{3}$. O objetivo, neste momento, é apresentar a evolução do uso da metodologia em um espaço preparado para a sua aplicação, o laboratório de voo, bem como o resultado de dois semestres de pesquisa sobre a percepção dos discentes do CSTPPA quanto ao seu aprendizado com a metodologia. A escolha da metodologia de aprendizagem baseada em cenário de simulação para a aprendizagem de alunos de um curso de pilotagem de aeronaves se deve ao uso constante de simuladores na aviação comercial e militar.

Pode-se dizer que a simulação é qualquer ambiente sintético ou artificial criado para gerenciar as experiências individuais ou de um grupo com a realidade (BELL, KANAR \& KOZLOWSKI, 2008, p. 5). Nesse sentido, a simulação ou a aprendizagem baseada em simulação com o uso da tecnologia é, por consequência, definida como qualquer prática em ambiente virtual criado para trabalhar, desenvolver competências e, com isso, aprimorar a capacidade de ação, atuação, execução do aprendiz (SALAS et al., 2009, p. 560).

No uso de simuladores na aviação, constata-se ainda que eles podem ser classificados, de uma forma geral, em simuladores de voo, ou melhor dizendo, Dispositivos de Treinamento Simuladores de Voo (FSTD - Flight Simulator Training Devices), de alta fidelidade e usados para treinar pilotos que já estão em linha aérea, e os simuladores de voo de baixa fidelidade, usados para o melhorar o ensino e aprendizagem dos pilotos que estão iniciando a carreira e ainda não possuem todas as licenças necessárias para trabalharem profissionalmente.

Seguindo esse raciocínio, os simuladores nos cursos superiores de tecnologia em pilotagem de voo no Brasil fazem uso de computadores de baixo custo como instrumento de aprendizagem no ensino acadêmico e são revestidos de uma abordagem pedagógica adequada a esse momento da formação. Os autores Garcia e Junior (2013) entendem que o desempenho e a aprendizagem dos alunos têm melhorado bastante com o uso das novas tecnologias que reproduzem o cenário do voo e aumentam o realismo. Sendo uma ferramenta de aprendizagem dinâmica, o cenário de simulação é fonte de motivação para o aluno que pode aplicar no voo e no simulador os conceitos apresentados pelo professor em sala de aula e dar sentido real e concreto a eles.

Nesse sentido, a metodologia de cenário, aliada ao uso desse dispositivo de treinamento de voo, busca a aplicação da teoria na prática de modo organizado em um currículo baseado em competências, tornando-se um desafio tanto para os alunos quanto para os professores. Apesar de não haver pesquisas conclusivas sobre a eficácia do uso de dispositivos de treinamento de voo de baixo custo na formação de pilotos de aeronaves (BELL; KANAR; KOZLOWSKI, 2008), a presente pesquisa conseguiu aplicar a teoria na prática em várias disciplinas e os discentes conseguiram verificar o quanto a sua aprendizagem cresceu nesse ambiente.

3 Vinculada ao projeto de pesquisa "Relações entre as Inovações Pedagógicas e a Aprendizagem Discente nos Cursos de Graduação da Universidade Tuiuti do Paraná, coordenado pelo Núcleo de Apoio Docente - NAD, com o objetivo de avaliar a aprendizagem dos estudantes da graduação da Universidade Tuiuti do Paraná, quando mediada por metodologias ativas e, ao mesmo tempo, promover a qualificação das práticas pedagógicas de docentes dos cursos de graduação da Universidade. 


\section{APRENDIZAGEM BASEADA EM CENÁRIO DE SIMULAÇÃO}

A aprendizagem baseada em cenário de simulação é, segundo Cox $(2010$, p. 20) um braço da metodologia baseada em problemas (PBL) e facilita a aprendizagem, o desenvolvimento e a transferência de habilidades de raciocínio. 0 uso da metodologia baseada em cenários pode ser aplicada desde o treinamento inicial do piloto até ao de manobras especiais, onde cenários são criados para que o piloto aprenda a gerenciar os recursos da cabine de voo, exercitando sua capacidade de julgar e tomar decisões, entre outras competências essenciais à atividade (COX, 2010).

Um dos estudos que tratam de competências no ensino superior é a de Kouwenhoven (2009), que analisa a educação baseada em competências através das descobertas das ciências cognitivas. Sua pesquisa conclui que o currículo baseado em competências tem uma abordagem construtivista e é orientado para a prática profissional do aluno e para o desenvolvimento das competências já adquiridas. Portanto, para o treinamento de novos pilotos, é muito bem aceito o uso de computadores de mesa para a simulação do voo e alguns estudos realizados sobre o assunto indicam sua eficiência, mesmo não tendo o realismo dos simuladores de alta precisão. (LINTERN, et al., 1990; LINTERN, et al., 1997; ORTIZ, 1994; DENNIS, HARRIS, 1998; TAYLOR, et al., 1999).

Para os autores Taylor e llif (1969), os simuladores de voo são úteis para o treinamento de caráter procedimental ou de voo por instrumentos e mostram bons resultados na aquisição de conhecimento. Confirmando essa ideia, os autores Vogel et. al. (2006) apontam que resultados de estudos empíricos acerca de simulações e jogos mostraram que, em todas as situações e variáveis, a aprendizagem das pessoas que utilizam jogos interativos ou simulações, quando comparados àquelas que usam métodos tradicionais de ensino, apresentam ganhos cognitivos significativamente mais altos e melhores.

Na formação dos alunos de pilotagem de aeronaves, a aprendizagem baseada em cenário de simulação é considerada como uma efetiva e dinâmica ferramenta de treinamento (ZANTOW; KNOWLTON; SHARP, 2005), que foi se aprimorando ao longo dos anos, passando a dar ênfase para a aprendizagem em que os alunos avaliam situações que imitam a realidade e devem reagir adequadamente ao momento do voo (FEDERAL AVIATION ADMINISTRATION, 2020, p. 7-12).

\section{MÉTODOS E TÉCNICAS}

O uso da metodologia de aprendizagem baseada em cenário de simulação no curso de pilotagem tem o objetivo de fundamentar a aplicação dessa metodologia de aprendizagem ativa, inovar na prática pedagógica e impactar na melhora da aprendizagem dos estudantes do ensino superior. Um ponto importante no uso da metodologia no CSTPPA foi a criação do laboratório de voo, que permite que o planejamento da aplicação da metodologia contemple três princípios fundamentais: a) a organização da disciplina em uma perspectiva interdisciplinar; b) a relação teoria e prática dos conteúdos da disciplina; c) a autonomia dos estudantes.

\subsection{O laboratório de simulação de voo e as disciplinas que o utilizam no Curso Superior de Tecnologia em Pilotagem Profissional de Aeronaves}

Sabe-se que a atividade de voar em uma aeronave é uma tarefa complexa e requer um grande nível de atenção por parte do piloto e, da mesma forma, treinamento constante. Para tanto, os dispositivos de treinamento de voo são utilizados como um ótimo recurso para diminuir o custo e os perigos que estão associados ao aprendizado do voo. (COX, 2010, p.4). Os simuladores oferecem um método seguro e econômico de treinamento de operadores e, segundo Ruiz, Aguado e Moreno (2014, p. 182), 
apresentam vários recursos que favorecem a aprendizagem de pilotos, tais como, exercitar a tomada de decisão, avaliar e prever potenciais soluções futuras. Ainda segundo os autores, o simulador permite que pessoas sejam colocadas em um cenário onde são criadas situações artificiais que podem ser adaptadas para o aprimoramento da experiência de aprendizagem.

No Curso Superior de Tecnologia em Pilotagem Profissional de Aeronaves, o uso do laboratório de voo é intenso, já que os docentes utilizam os recursos do software instalado nos computadores nas cabines de voo para treinar conteúdos aprendidos durantes as aulas de navegação aérea, teoria de voo, tráfego aéreo internacional, inglês II, além dos projetos interdisciplinares e das práticas laboratoriais. A montagem desse laboratório aconteceu no final de 2013, e o programa chamado Microsoft Flight Simulator X (FSX) foi instalado por ser capaz de criar vários tipos de cenários, permitindo ao docente adequar a sua aula e engajar o aluno de maneira ativa no processo da aprendizagem.

Como é apresentado na figura 1, o espaço de aprendizagem foi montado com 20 estações, para os alunos praticarem as missões que lhe são dadas. Essas estações são compostas com computadores de baixo custo, joysticks e fones de ouvido. Outras cinco estações também estão nesse espaço e são destinadas para os Órgãos Operacionais Controle de Tráfego e Solo, Torre de Controle, Centro de Controle de Aproximação (APP) e Centro de Controle de Área (ACC), igualmente equipadas com fones de ouvidos. A configuração das telas dos computadores que simulam o controle de tráfego aéreo é bastante semelhante ao real.

Figura 1 - Layout do laboratório de simulação de vôo.

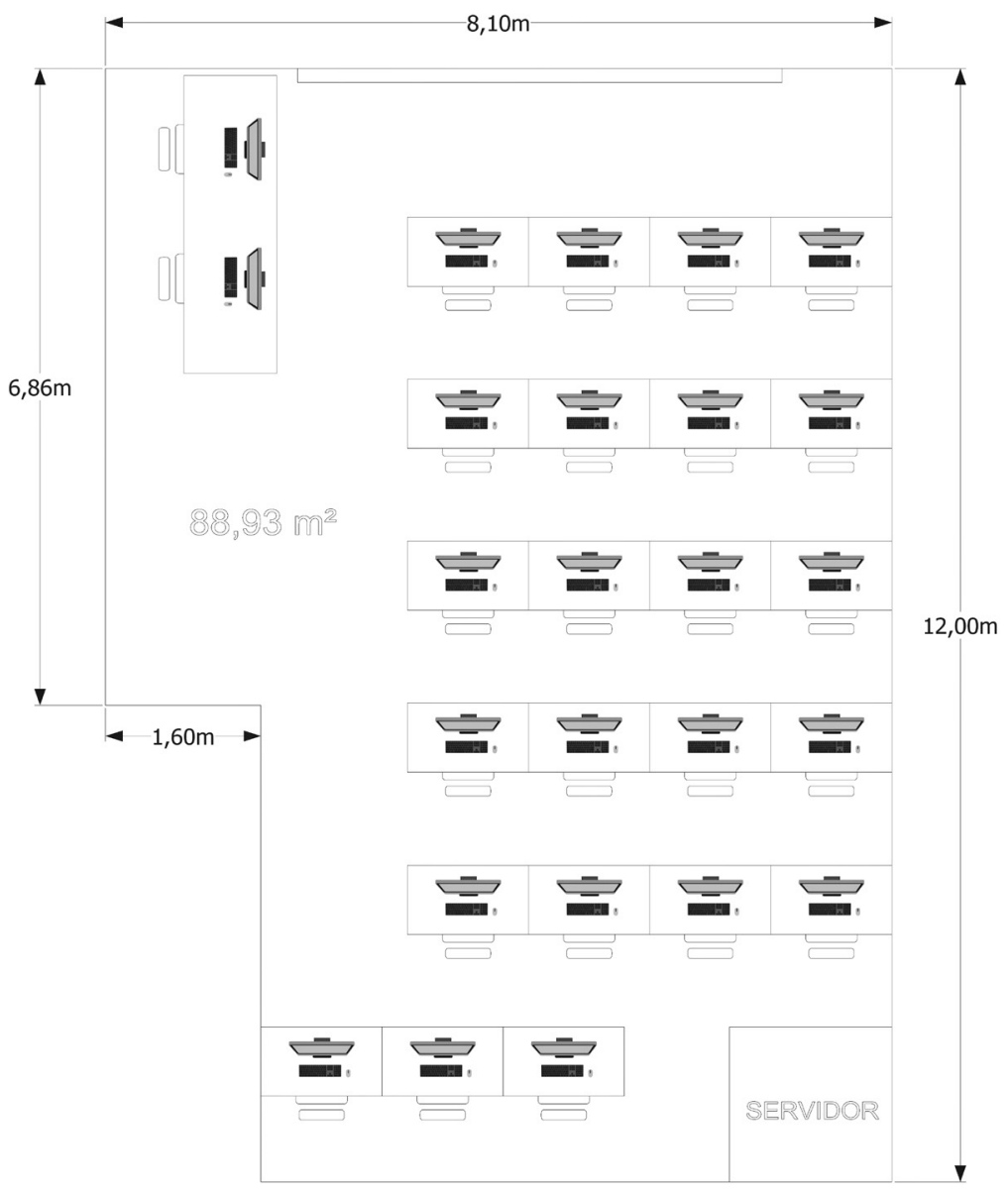

Fonte: Manual do Laboratório de Voo do CSTPPA 
No cenário, o aluno pode vivenciar o aprendizado pela experiência, ou seja, ele aprende fazendo, pensa sobre o que está aprendendo e assimila o que foi aprendido. Outro aspecto importante na aprendizagem com simuladores é a estruturação das sessões, as quais devem ter objetivos de aprendizagem bem definidos para passar pelos estágios do ciclo de experiência de maneira lógica e estruturada, e combinar a experiência ativa no simulador com uma subsequente análise e reflexão do que foi aprendido (FANNING; GABA, 2007, p. 1).

\section{RESULTADOS}

\subsection{O uso do laboratório de voo}

Nessa etapa da pesquisa, avaliou-se o aumento do uso do laboratório pelos docentes e a percepção dos alunos da melhoria do seu conhecimento com a aplicação da metodologia de aprendizagem baseada em cenários. Pode-se perceber, de acordo com o Gráfico 1, que o uso do laboratório de voo foi crescendo ao longo de seis anos.

Gráfico 1 - Histórico das disciplinas que utilizava o laboratório de vôo.

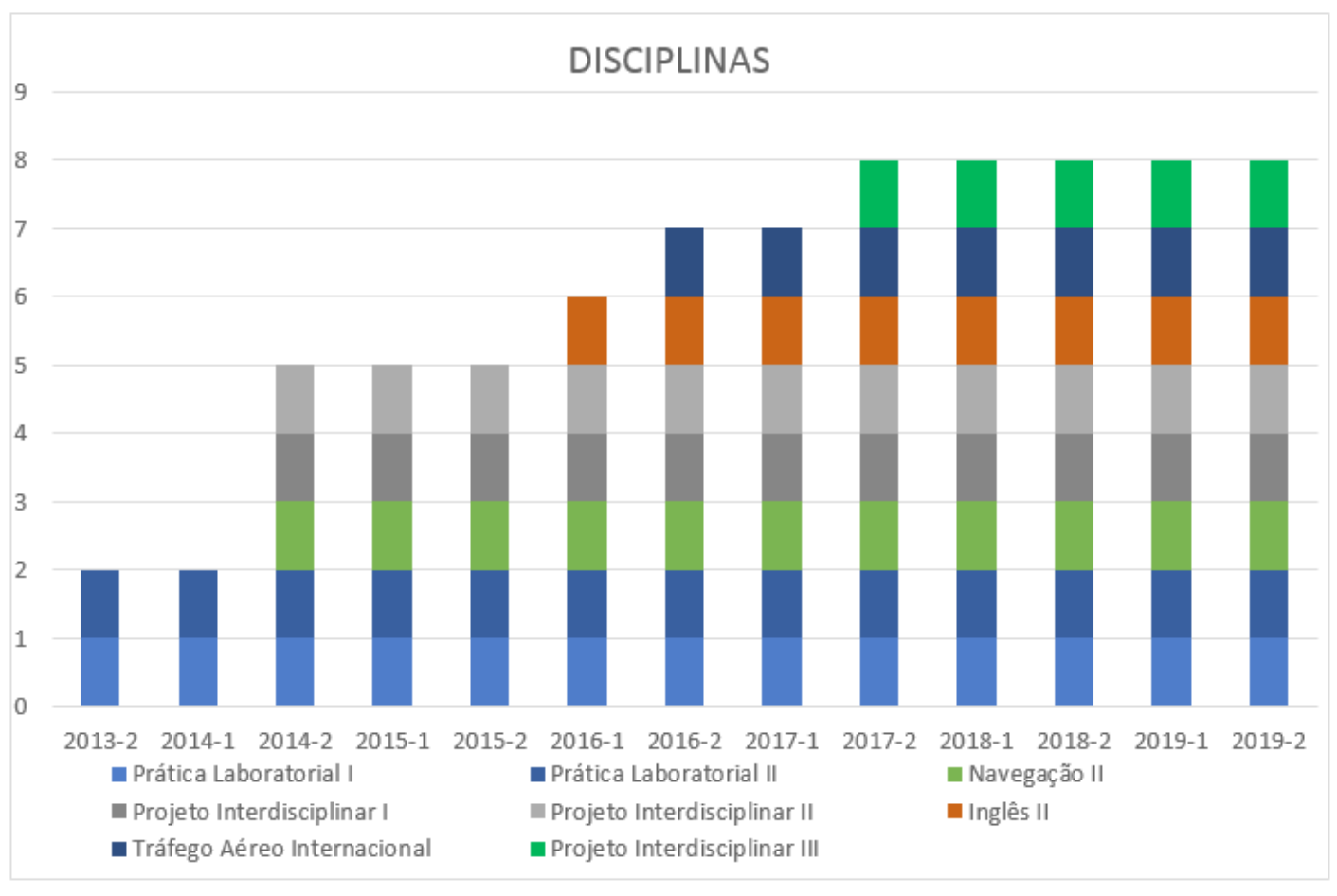

Fonte: Autores.

Em 2013, apenas duas disciplinas utilizavam o laboratório e, um ano depois, quatro disciplinas incluíram aulas práticas no seu planejamento. De 2017 até a data atual, as disciplinas que utilizam o laboratório de voo são em número de oito, e dos quatro períodos que compõem o curso, apenas no primeiro período não há disciplinas utilizando a metodologia de cenário de simulação. 0 gráfico 2 apresenta a quantidade de horas-aula ministradas no laboratório por semestre, desde a sua criação. 
Gráfico 2 - Quantidade de horas-aula ministradas no Laboratório de Simulação de Voo de 2013-2 a 2019-1 e previsão para 2019-2 de acordo com as turmas existentes.

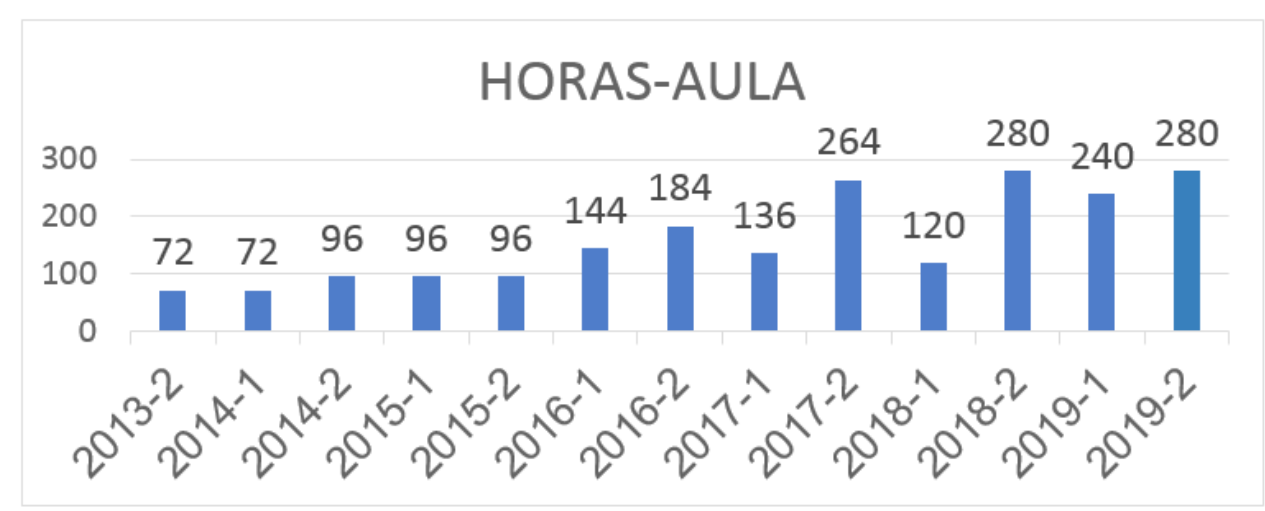

Fonte: Autores.

Já no Quadro 1, as horas-aula ministradas pelos docentes são apresentadas por disciplina.

Quadro 1 - Horas-aula ministradas no Laboratório de 2013-2 a 2019-1 e previsão para 2019-2.

\begin{tabular}{|l|l|l|l|l|l|l|l|l|l|}
\hline & $\begin{array}{l}\text { PL 1 } \\
\text { Prática } \\
\text { Laboratorial I }\end{array}$ & $\begin{array}{l}\text { PL 2 } \\
\text { Prática } \\
\text { Laboratorial II }\end{array}$ & $\begin{array}{l}\text { NAV 2 } \\
\text { Navegação } \\
\text { Aérea II }\end{array}$ & $\begin{array}{l}\text { PI 1 } \\
\text { Projeto } \\
\text { Interdisciplinar I }\end{array}$ & $\begin{array}{l}\text { PI 2 } \\
\text { Projeto } \\
\text { Interdisciplinar II }\end{array}$ & $\begin{array}{l}\text { ING 2 } \\
\text { Inglês } \\
\text { Aplicado II }\end{array}$ & $\begin{array}{l}\text { TAI } \\
\text { Tráfego Aéreo } \\
\text { Internacional }\end{array}$ & $\begin{array}{l}\text { PI 3 } \\
\text { Projeto } \\
\text { Interdisciplinar III }\end{array}$ & $\begin{array}{l}\text { Total de Horas- } \\
\text { aula Semestrais }\end{array}$ \\
\hline $2013-2$ & 36 & 36 & & & & & & & 72 \\
\hline $2014-1$ & 36 & 36 & & & & & & & 72 \\
\hline $2014-2$ & 36 & 36 & 8 & 8 & 8 & & & & 96 \\
\hline $2015-1$ & 36 & 36 & 8 & 8 & 8 & & & & 96 \\
\hline $2015-2$ & 36 & 36 & 8 & 8 & 8 & & & & 96 \\
\hline $2016-1$ & 40 & 40 & 8 & 8 & 8 & 40 & & & 144 \\
\hline $2016-2$ & 40 & 40 & 8 & 8 & 8 & 40 & 40 & & 184 \\
\hline $2017-1$ & & 40 & 8 & & 8 & 40 & 40 & & 264 \\
\hline $2017-2$ & 40 & 40 & 8 & 8 & 8 & 40 & 40 & & 80 \\
\hline $2018-1$ & 40 & & 16 & 8 & 16 & 40 & & & 120 \\
\hline $2018-2$ & 40 & 40 & 16 & 8 & 16 & 40 & 40 & 80 & 280 \\
\hline $2019-1$ & 40 & 40 & 16 & 8 & 16 & 40 & 40 & 40 & 240 \\
\hline $2019-2$ & 40 & 40 & 16 & 8 & 16 & 40 & 40 & 80 & 280 \\
\hline
\end{tabular}

Fonte: Autores.

Assim, inicia-se o uso do laboratório de simulação de voo a partir do segundo período com duas disciplinas, Projeto Interdisciplinar I e Prática Laboratorial I. No terceiro período, os alunos aplicam no laboratório de simulação de voo os conteúdos das disciplinas Navegação Aérea II, Inglês Aeronáutico II e Projeto Interdisciplinar II. Por fim, no quarto período, utilizam o laboratório de simulação de voo nas disciplinas Tráfego Aéreo Internacional, Prática Laboratorial II e Projeto Interdisciplinar III. Essas disciplinas trabalham de forma interdisciplinar com todas as demais que compõem o curso e inserem o aluno em situações que ele necessita buscar o conhecimento adquirido nas aulas teóricas para resolver os problemas apresentados no voo. Percebe-se, portanto, o aumento significativo das disciplinas, 0 que significa que os docentes aderiram a ideia de utilizar a metodologia de cenário no laboratório de voo para promover a melhora do aprendizado. 


\subsection{Percepção dos discentes sobre o uso do laboratório de voo}

Além de avaliar o aumento do uso do laboratório de voo no CSTPPA, também faz parte do estudo saber qual foi a percepção do aluno do Curso Superior de Pilotagem de Aeronaves da Universidade Tuiuti do Paraná sobre sua aprendizagem e atuação nos cenários simulados durante os dois semestres de 2019. Elaborou-se um questionário eletrônico com 16 questões, 14 objetivas e duas dissertativas, que foi aplicado nas duas últimas semanas dos semestres com os alunos matriculados nas disciplinas acima elencadas, do segundo, terceiro e quarto períodos. Os gráficos 3 e 4 apresentam as disciplinas e o número de alunos matriculados em 2019 e o número de respostas por turma e período. Já as Quadros 2 e 3 , mostram as disciplinas e respostas por período letivo.

Gráfico 3 - Alunos que responderam ao questionário Forms em 2019/1

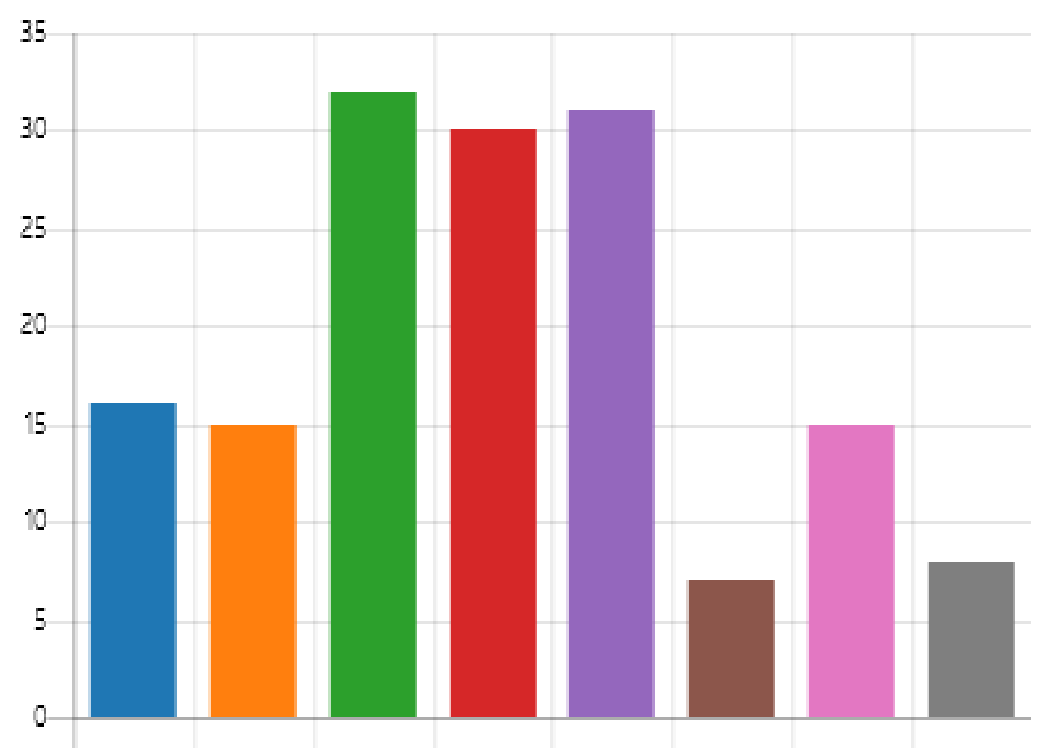

Fonte: Autores.

Quadro 2 - Disciplinas e número de alunos em 2019/1.

\begin{tabular}{|l|l|l|}
\hline \multirow{2}{*}{$\begin{array}{l}\text { 2. PERÍODO } \\
\text { NOITE }\end{array}$} & Prática Laboratorial I & -16 \\
\cline { 2 - 3 } & Projeto Interdisciplinar I & -15 \\
\hline \multirow{3}{*}{$\begin{array}{l}\text { 3. PERÍODO } \\
\text { MANHÃ E NOITE }\end{array}$} & Inglês II & -32 \\
\cline { 2 - 3 } & Navegação II & -30 \\
\cline { 2 - 3 } & Projeto Interdisciplinar II & -31 \\
\hline \multirow{3}{*}{$\begin{array}{l}4^{\circ} \text { PERÍODO } \\
\text { NOITE }\end{array}$} & Tráfego Aéreo Internacional & -7 \\
\cline { 2 - 3 } & Prática Laboratorial II & -15 \\
\cline { 2 - 3 } & Projeto Interdisciplinar III & -8 \\
\hline
\end{tabular}

Fonte: Autores. 
Gráfico 4 - Alunos que responderam ao questionário Forms em 2019/2.

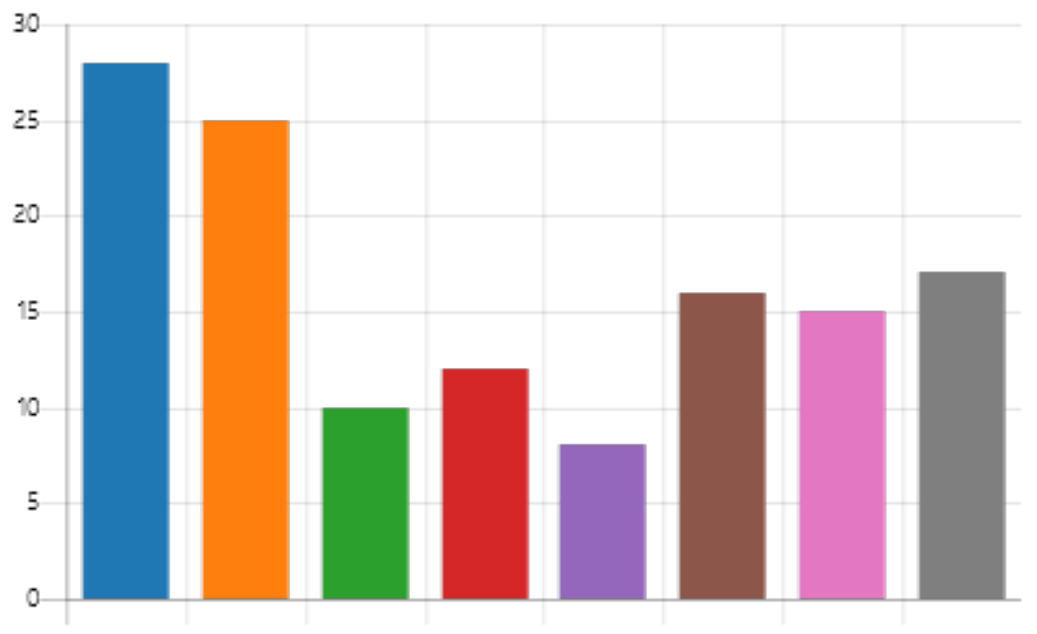

Fonte: Autores.

Quadro 3 - Disciplinas e número de alunos em 2019/2

\begin{tabular}{|c|c|c|}
\hline \multirow{2}{*}{$\begin{array}{l}2^{\circ} \text { PERÍODO } \\
\text { NOITE }\end{array}$} & Prática Laboratorial I & -28 \\
\hline & Projeto Interdisciplinar I & -25 \\
\hline \multirow{3}{*}{$\begin{array}{l}3^{\circ} \text { PERÍODO } \\
\text { MANHÃ E NOITE }\end{array}$} & Inglês II & -10 \\
\hline & Navegação II & -12 \\
\hline & Projeto Interdisciplinar II & -08 \\
\hline \multirow{3}{*}{$\begin{array}{l}4^{\circ} \text { PERÍODO } \\
\text { NOITE }\end{array}$} & Tráfego Aéreo Internacional & -16 \\
\hline & Prática Laboratorial II & -15 \\
\hline & Projeto Interdisciplinar III & -17 \\
\hline
\end{tabular}

Fonte: Autores.

A análise das respostas dos alunos quanto ao uso do laboratório de simulação de voo, em ambos os períodos pesquisados, demonstra que mais de $80 \%$ dos discentes percebem que há, no processo de aprendizagem, uma grande relação existente entre a teoria estudada e a prática da simulação e, com isso, há melhora do aprendizado. Foi apontado também que há promoção de um aprendizado mais efetivo, quando comparado com a metodologia tradicional (aula expositiva). As respostas mostram também que a maioria dos alunos se sentiu mais motivada para estudar, facilitando a fixação do conteúdo estudado. Os alunos notaram ainda ser imprescindível a utilização dos conteúdos das demais disciplinas nas aulas de simulação, o que aumentava a exigência da própria responsabilidade por sua aprendizagem.

Com relação ao desenvolvimento das principais competências pela metodologia de simulação, os discentes apontaram para o aumento da compreensão dos conteúdos e da rapidez de raciocínio. Também foram destacadas a organização na resolução de problemas e relação de ideias, e o aumento de responsabilidade e trabalho em equipe.

Sobre as três vantagens mais relevantes observadas com a prática da simulação, aparece, em primeiro lugar, a capacidade de perceber a relação entre teoria e prática; em segundo, a fixação do conteúdo e a vivência prática profissional e, logo em seguida, a visão sistêmica do processo e a autonomia do aluno sobre o seu aprendizado. Sobre as três desvantagens mais relevantes observadas com a prática da simulação, a maioria apontou não haver alguma e, em segundo lugar, surge a necessidade de se ter conhecimento prévio. 
A análise das respostas dos discentes aponta ainda quais são as principais dificuldades encontradas na realização da simulação, os aspectos de relevância, a diferença de nível de conhecimento no uso do programa de voo instalado nos computadores, até a falta do computador em casa para treinar. Sobre o que os alunos sugerem para melhorar a prática da simulação e a aprendizagem, foi apontado o aumento de aulas no laboratório de voo com sugestão de maior frequência.

\section{CONCLUSÃo}

A pesquisa do grupo de estudos do Curso Superior de Tecnologia em Pilotagem Profissional de Aeronaves sobre a metodologia de aprendizagem baseada em cenário de simulação, ligada ao projeto de pesquisa sobre metodologias inovadoras de aprendizagem desenvolvido na Universidade Tuiuti do Paraná se propôs a aplicar a metodologia ativa, utilizando o laboratório de voo, e a elaborar os cenários necessários para a aplicação do conhecimento teórico ministrado em sala de aula. Para tanto, foi necessário o aprofundamento da sua aplicação, assim como delimitar os conceitos e as áreas de atuação, já que o processo de ensino-aprendizagem no ensino superior com a utilização de metodologias inovadoras ganha relevância para o docente contemporâneo.

A escolha da metodologia de aprendizagem baseada em cenário de simulação se deu em função do seu uso na formação de pilotos de aeronaves, pois é essencial para a verificação da eficácia e eficiência das práticas docente nesse processo. Assim, quando bem planejada, o uso da metodologia de cenário de simulação se torna uma prática exitosa e as disciplinas que fazem uso dessa metodologia conseguem motivar os alunos a aprender os conteúdos teóricos, já que são aplicados em um ambiente que exige deles o preparo prévio do que foi ensinado. Essa metodologia também facilita o desenvolvimento das competências que envolvem a profissão de piloto.

Tendo o conhecimento adequado da metodologia, foi dada a continuidade da pesquisa e a segunda etapa ocorreu nos dois semestres de 2019, em que se fez o levantamento do uso do laboratório de voo desde a sua criação e apurou-se a percepção dos estudantes dos três períodos que utilizam o espaço sobre a relação entre a melhora do seu aprendizado e a metodologia baseada em cenário de simulação. Na análise dos dados coletados pelo instrumento de pesquisa elaborado sobre a percepção dos alunos, identificou-se os aspectos positivos e negativos da metodologia de aprendizagem baseada em cenário de simulação.

Acredita-se que o processo de ensino-aprendizagem no ensino superior com a utilização de metodologias ativas ganha relevância para o aluno, quando ele percebe o quanto aprimora o seu aprendizado. Ainda, a percepção dos discentes motiva, no contexto do docente contemporâneo na atividade aérea, a verificação da eficácia e eficiência das práticas docente nesses processos e o seu aprimoramento. 0 próximo passo da pesquisa se volta para o aperfeiçoamento da metodologia, para que o aluno tenha uma aprendizagem ainda mais eficaz. 


\section{REFERÊNCIAS}

BELL, Bradford S.; KANAR, Adam M.; KOZLOWSKI, Steve W. J. Current issues and future directions in simulation-based training in North America. Cornell University, 2008. Disponível em: http://digitalcommons. ilr.cornell.edu/articles/412/. Acesso em: 14 mar. 2019.

COX, Brenda A. Scenario Based Training in an Aviation Training Environment. All Regis University Theses. Paper 2. 2010. Disponível em: https://pdfs. semanticscholar.org/36b4/467e8dfb9f6327cdff4a2b4ffcfbb9e3361c. pdf. Acesso em: 5 jul. 2018.

DENNIS, Kerry A.; HARRIS, Don. Computer-based simulation as an adjunct to ab initio flight training. In: The International Journal of Aviation Psychology, 8(3), 277-292. 1998. Disponível em: https://www. tandfonline.com/doi/abs/10.1207/s15327108ijap0803_6. Acesso em: 23 jul. 2018.

FANNING, Ruth M.; GABA, David M. The Role of Debriefing in Simulation-Based Learning. Simulation in Healthcare, Volume 2, Number 1, Spring 2007. Disponível em: https://journals.Iww.com/ simulationinhealthcare/Fulltext/2007/00220/The_Role_of_Debriefing_in_Simulation_Based.7.aspx. Acesso em: 23 jul. 2018.

FEDERAL AVIATION ADMINISTRATION. Aviation Instructor's Handbook. FAA-H-8083-9. U.S. Department of Transportation. Federal Aviation Administration. Flight Standards Service, 2020. Disponível em: https://www.faa.gov/regulations_policies/handbooks_manuals/aviation/aviation_instructors_handbook/ media/aviation_instructors_handbook.pdf. Acesso em: 11 ago. 2020.

KOUWENHOVEN, Wim. Competence-based curriculum development in higher education: a globalised concept? In: Technology education and development. Publisher: Intech, 2009. Disponível em: https:// www.researchgate.net/publication/227452769 Competency Based Curriculum in Higher Education A Necessity_Grounded_by_Globalization_English_version/citation/download. Acesso em: 10 nov. 2018.

LINTERN, Gavan; ROSCOE, Stanley N.; KOONCE, Jefferson M.; SEGAL, Leon D. Transfer of landing skills in beginning flight training. Human Factors, 32, 319-327. 1990. Disponível em: https://journals.sagepub. com/doi/abs/10.1177/001872089003200305. Acesso em: 20 out. 2018.

LINTERN, Gavan; TAYLOR, Henry L.; KOONCE, Jefferson M.; KAISER, Robert H.; MORRISON, Gregory A. Transfer and quasi-transfer effects of scene detail and visual augmentation in landing training. In: The International Journal of Aviation Psychology, 7(2), 149-169. 1997. Disponível em: https://journals. sagepub.com/doi/abs/10.1177/001872089003200305. Acesso em: 28 out. 2018.

ORTIZ, Gustavo A. Effectiveness of PC-based flight simulation. In: The International Journal of Aviation Psychology, 4(3), 285-291. 1994. Disponível em: https://www.tandfonline.com/doi/abs/10.1207/ s15327108ijap0403_5. Acesso em: 28 out. 2018.

RUIZ, Sergio; AGUADO, Carlos E.; MORENO, Romualdo. Educational Simulation in Practice: A Teaching Experience Using a Flight Simulator. In: Journal of Technology and Science Education, vol. 3, p. 181200, Barcelona, Espanha, 2014. Disponível em: https://www.jotse.org/index.php/jotse/article/view/129. Acesso em: 20 out. 2019.

SALAS, Eduardo; WILDMAN, Jessica L.; PICCOLO, Ronald F. Using Simulation-Based Training to Enhance Management Education. Academy of Management Learning \& Education, 2009, Vol. 8, No. 4, 559-573. 
Disponível em: https://www.researchgate.net/publication/325008015_Using_Simulation-Based_ Training_to_Enhance_Management_Education. Acesso em: 28 out. 2018.

TAYLOR, Henry L.; LINTERN, Gavan; HULIN, Charles L.; TALLEUR, Donald A.; EMANUEL, Tom; PHILLIPS, Sibel. Transfer of training effectiveness of a personal computer aviation training device. In: The International Journal of Aviation Psychology, volume 9, issue 4, 1999. Disponível em: https://www. researchgate.net/publication/235146364_Transfer_of_Training_Effectiveness_of_Personal_ComputerBased_Aviation_Training_Devices. Acesso em: 20 out. 2018.

TAYLOR, Lawrence W., Junior; ILIFF, Kenneth. W. Fixed-base simulator pilot rating surveys for predicting lateral-directional handling qualities and pilot rating variability. In: Report Number: NASA-TN-D-5358. Edwards, CA: NASA Dryden Flight Research Center, 1969. Disponível em: https://www.nasa.gov/centers/ dryden/pdf/87769main_H-626.pdf. Acesso em: 20 out. 2018.

VOGEL, Jennifer; VOGEL, David; CANNON-BOWERS, Jan; BOWERS, Clint; MUSE, Kathryn; WRIGHT, Michelle. Computer gaming and interactive simulations for learning: A meta-analysis. In: Educational Computing Research, Vol. 34(3) 229-243. 2006. Disponível em: https://www.researchgate.net/profile/ Michelle_Wright3/publication/233742169_Computer_gaming_and_interactive_simulations_for_learning_A_ meta-analysis/links/0912f50be3158c6f20000000.pdf. Acesso em: 24 out. 2018.

ZANTOW, Kenneth; KNOWLTON, Dave; SHARP, D. More Than Fun and Games: Reconsidering the Virtues of Strategic Management Simulations. In: Academy of Management Learning and Education, The. 4. 10.5465/ AMLE.2005.19086786. 2005 Disponível em: https://www.researchgate.net/publication/277826746 More_Than_Fun_and_Games_Reconsidering_the_Virtues_of_Strategic_M. Acesso em: 20 nov. 2019. 